\title{
Déterminisme de la polarité dorso-ventrale de l'embryon de drosophile
}

Éliane Mohier

\section{ADRESSE}

E. Mohier : directeur de recherche au Cnrs. Institut Jacques-Monod, Cnrs et université Paris VII, 2, place Jussieu, 75251 Paris
Le développement précoce est l'objet de nombreuses études chez divers organismes. Chez la drosophile, les possibilités offertes par la génétique, les expériences de transplantation de cytoplasme ainsi que les données récentes de la biologie moléculaire ont permis d'élucider tout ou partie des mécanismes responsables de la mise en place de la polarité embryonnaire. Un nombre restreint de gènes, pour la plupart maternels, sont impliqués dans ce processus et contrôlent quatre systèmes indépendants - trois pour l'axe antéro-postérieur (A/P), et un pour l'axe dorso-ventral $(\mathrm{D} / \mathrm{V})$ - qui déterminent les différents territoires de l'embryon par l'intermédiaire de produits spécifiquement localisés dans l'œuf. Cette revue considère plus précisément l'établissement de la polarité dorso-ventrale, responsable de la mise en place des feuillets embryonnaires. Le développement dorsoventral est contrôlé par un seul système de gènes maternels, les gènes du groupe dorsal. Ce groupe comprend 11 gènes dont la perte de fonction aboutit à un développement dorsalisé, tandis qu'un douzième gène, cactus, lorsqu'il est muté, conduit à un développement ventralisé. Ces gènes ont pu être ordonnés selon une hiérarchie de fonctions qui concourt à la formation d'un grandient de concentration nucléaire du produit du gène dorsal. Le produit dosal correspond au morphogène $\mathrm{D} / \mathrm{V}$ et présente une forte homologie avec le facteur de transcription NF- $\kappa$ B. Parmi les 11 gènes du groupe dorsal, trois sont requis dans la lignée somatique, ce qui suggère l'existence d'un mécanisme de transduction d'un signal émis de façon asymétrique par les cellules folliculaires et qui, par l'intermédiaire d'un récepteur transmembranaire, activerait spécifiquement la translocation nucléaire du produit dorsal. Ce modèle, fondé sur des résultats récents, met en lumière l'importance de la polarité de la chambre ovarienne dans le déterminisme de la polarité du futur embryon et, dans ce sens, accorde une importance déterminante au noyau de l'ovocyte, dont la localisation asymétrique servirait de point de départ à la mise en place de la polarité dorso-ventrale. 
$\mathbf{L}$

'une des ambitions des biologistes est de comprendre comment un organisme sc reproduit, c'est-à-dirc transmet ses caractéristiques d'une génération à la suivante. Ce cycle s'effectue selon unc alternance de formes simples (l'œuf) ct de formes complexes (l'organisme adulte). Sans aller jusqu'à prétendre répondre à la délicate question de l'ordrc d'apparition - Qui de l'œuf ou de la poule... ? - , le problème réside dans la connaissance de la complexité réelle de l'œuf. De quelle façon et dans quelle mesure le plan de l'animal adulte est-il contenu dans l'organisation d'une simple cellulc, l'œuf ? et de quelle façon cette information sera-t-elle interprétée pour aboutir à la réalisation de l'embryon? Très vite, la réponse à cette question qui peut se résumer par la formule : comment un œuf est-il capable de former un adulte ? - débouche sur une autre question : comment l'adulte est-il capable de fabriquer un œuf?

Notre compréhension des mécanismes morphogénétiques mis en jeu au cours du développement embryonnaire s'est considérablement enrichie $\mathrm{au}$ cours de la dernière décennie grâce à l'utilisation du modèle drosophile. L'approche génétique a non seulement permis d'identifier la quasi-totalité des gènes qui jouent un rôle spécifique au cours du développement précoce, mais encore, associée à l'embryologie classique et à la biologie moléculaire, elle a permis d'intégrer ces résultats en un schéma cohérent de l'établissement du plan d'organisation de l'embryon au cours du développement précoce.

Cette revue se propose de faire le point de l'état actuel de nos connaissances sur l'établissement de la polarité de l'embryon de drosophile, succinctement pour l'axe antéropostérieur (A/P) et de façon plus détaillée pour l'axe dorso-ventral $(\mathrm{D} / \mathrm{V})$. Les résultats rapportés proviennent de différents laboratoires dans le monde, mais il faut plus particulièrement citer le rôle essentiel qu'ont joué C. Nüsslein-Volhard (Tübingen, Allemagne) et les membres de son équipe (passés ou présents) dans les progrès réalisés dans ce domaine.

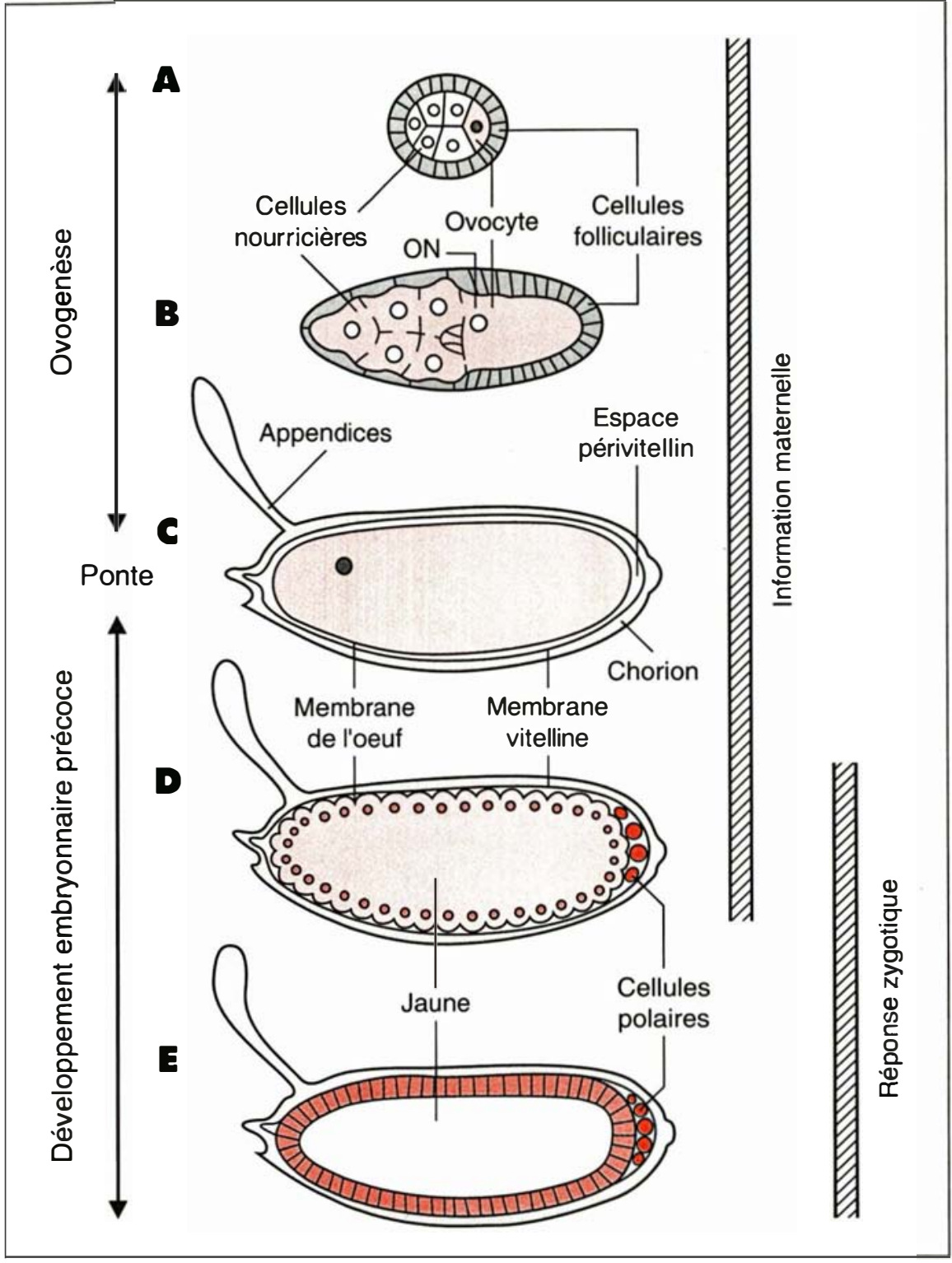

Figure 1. Schéma de l'ovogenèse et du développement précoce. (A) Chambre ovarienne précoce (stades 1 à 8 de l'ovogenèse). Le complexe cellules nourricières-ovocyte d'origine germinale est entouré d'une monocouche de cellules folliculaires d'origine somatique; l'ovocyte est toujours situé au pôle postérieur de la chambre ovarienne. (B) Chambre ovarienne stade 10 ; le volume de l'ovocyte est équivalent à celui de l'ensemble des cellules nourricières. Noter la position asymétrique du noyau de l'ovocyte (ON) dans la future région antéro-dorsale de l'œuf. (C) CEuf fraîchement pondu. II possède une polarité $A / P$ et $D N$ avec un dos plat et un ventre arrondi; outre sa membrane, cet œuf est entouré d'une membrane vitelline et d'un chorion lenveloppe rigide, protectrice) synthétisés par les cellules folliculaires. La membrane vitelline et la membrane de l'œuf définissant l'espace périvitellin, visible aux seules extrémités de l'œuf. (D) Stade du blastoderme syncitial. Les noyaux du syncitium ont migré vers la périphérie de l'œuf tandis que quelques cellules sont formées au pôle postérieur: les cellules polaires, qui sont les précurseurs de la lignée germinale. C'est à ce stade que commence l'activité transcriptionnelle du zygote. (E) Blastoderme cellulaire, constitué d'une monocouche d'environ 6000 cellules morphologiquement identiques. 


\section{RÉFÉRENCES}

1. Nüsslcin-Volhard C. Determination of the embryonic axes of Drosophila. Development $1991 ; 1$ (suppl) : 1-10.

2. St Johnston D, Nüsslcin-Volhard C. The origin of pattern and polarity in the Drosophila cmbryo. Cell 1992 ; 68 : 201-19.

3. Anderson KV, Nüsslcin-Volhard C. Information of the dorsoventral pattern of the Drosophila embryo is storcd as maternal mRNA. Nature 1984, 311: 223-7.

4. Roth S, Hiromi Y, Godt D, NüsslcinVolhard C. Cactus, a maternal gene required for the proper formation of the dorsoventral morphogen gradient in Drosophila cmbryos. Development 1991; 112 : 371-88.

5. Nüsslein-Volhard C. A dorsoventral shift of embryonic primordia in a new maternal effect mutant of Drosophila. Nature 1980 ; 283 : 474-6.

6. Steward R. Relocalization of the dorsal protein from the cytoplasm to the nuclcus correlates with its function. Cell $1989 ; 59$ : 1179-88.

7. Roth S, Stcin B, Nüsslcin-Volhard C. A gradient of nuclear localization of the dorsal protein determines dorsoventral pattern in the Drosophila cmbryo. Cell 1989; 59 1189-202.

8. Stcward R. Dorsal, an embryonic polarity gene in Drosophila, is homologous to the vertcbrate proto-oncogenc, c-rel. Science $1987 ; 238: 692-4$.

9. Ghosh S, Gifford A, Rivicre L, Tempst $P$, Nolan G, Baltimore D. Cloning of the p50 DNA binding subunit of NF $\kappa \mathrm{B}$ : homology to rel and dorsal. Cell $1990 ; 62$ : 1019-29.

10. Santamaria $P$, Nüsslcin-Volhard C Partial rescues of dorsal, a maternal effect mutation affecting the dorsoventral pattern of the Drosophila cmbryo by the injection of wild-type cytoplasm. EMBO J $1983 ; 2$ : 1695-9.

11. Anderson K, Bokla L, Nüsslein-Volhard C. Establishment of dorsal-ventral polarity in the Drosophila embryo : the induction of polarity by the Toll gene product. Cell $1985 ; 42$ : $791-8$

12. Hashimoto $\mathrm{C}$, Hudson $\mathrm{K}$, Anderson $\mathrm{K}$. The Toll gene of Drosophila, required for dorsal-ventral embryonic polarity, appears to cncode a transmembranc protein. Cell 1988 ; 52 : 269-79.
Avant d'aborder le développement $\mathrm{D} / \mathrm{V}$ qui fait l'objet de cette revue, un bref résumé du développement de la drosophile s'impose ainsi qu'une rapide synthèse des résultats connus à ce jour pour le développement $\mathrm{A} / \mathrm{P}$.

\section{Mise en place de la polarité antéro-postérieure}

La construction de l'ovocyte de drosophile fait appel à des types cellulaires d'origines différentes. Tout commence par une cellule souche de la lignée germinale qui, par suite de quatre divisions mitotiques, donne naissance à un groupe de 16 cellules sœurs qui communiquent entre elles par des ponts cytoplasmiques. Très tôt, l'une de ces cellules se différencie en ovocyte tandis que les 15 autres constituent les cellules nourricières. L'ensemble, cellules nourricières et ovocyte, est entouré d'un épithélium d'origine somatique, les cellules folliculaires, et constitue la chambre ovarienne. C'est à l'intérieur de la chambre ovarienne que se déroulent les différentes étapes de maturation qui aboutissent à la formation de l'œuf (figure 1, p. 655). Au cours de l'ovogenèse, les cellules nourricières sont très actives et produisent la plupart des ARN et des protéines qui seront déversés dans l'ovocyte vers la fin de l'ovogenèse. D'autres substances sont transportées par l'hémolymphe à travers les cellules folliculaires et également déposées dans un ovocyte qui, lui-même, semble transcriptionnellement inactif. Les cellules folliculaires synthétisent les enveloppes de l'ovocyte : la membrane vitelline et le chorion. Notons que l'œuf, lorsqu'il est pondu, possède clairement une asymétrie antéropostérieure et dorso-ventrale (figure $1 C$ ). Après la fécondation qui a lieu au moment de la ponte, le noyau du zygote se divise rapidement pour former un syncitium. Vers la $12^{\mathrm{e}}$ division, la quasi-totalité des noyaux migrent vers le cortex de l'œuf, c'est le blastoderme syncitial (figure 1D). Après deux divisions supplémentaires, il y a cellularisation et formation du blastoderme cellulaire (figure 1E). C'est à l'étape du blastoderme syncitial que commence la transcription des gènes du zygote, tout ce qui précède étant sous le contrôle d'une information maternelle.

Le blastoderme constitue une étape cruciale du développement embryonnaire. Non seulement c'est à ce stade qu'a lieu le relais entre l'information maternelle et l'information zygotique, mais aussi le plan d'organisation de l'embryon est achevé, et chaque cellule, bien que morphologiquement indistinguable des autres, est engagée dans une voie de développement qui dépend exclusivement de sa position par rapport aux axes A/P et D/V. Ces restrictions des potentialités de développement ont permis d'établir des cartes des territoires présomptifs au stade du blastoderme. Ces territoires sont au nombre de sept pour l'axe A/P et de quatre pour l'axe $\mathrm{D} / \mathrm{V}$ (figure 2).

Étudié depuis plus longtemps, le développement $\mathrm{A} / \mathrm{P}$ est actuellement le mieux connu, et cela en dépit de sa plus grande complexité. En effet, il est apparu que la mise en place de la polarité $\mathrm{A} / \mathrm{P}$ fait intervenir trois systèmes indépendants $[1,2]$.

Le premier système, " antérieur ", est responsable de la formation des régions segmentées de la tête et du thorax. Le deuxième système " postérieur ", définit la région segmentée abdominale, tandis que le troisième système, dit des " extrémités ", contrôle les régions non segmentées antérieures et postérieures, respectivement, l'acron et le telson. Bien que pour une large mesure indépendants, ces trois systèmes partagent un certain nombre de points communs : (1) dans tous les cas, le produit d'un gène est localisé dès l'ovogenèse en un site spécifique de l'ovocyte et fonctionne comme information de position ; (2) pour chaque système, cette information de position a pour conséquence la distribution asymétrique d'un produit de gène qui fonctionne comme un facteur de transcription ; (3) ce facteur de transcription, distribué en un gradient de concentration, définit des zones d'expression d'un ou de plusieurs gènes zygotiques cibles.

Ces principes, énoncés par C. Nüsslein-Volhard, bien que globalement vérifiés, souffrent quelques variantes à l'intérieur de chaque système.

Ainsi, pour le système antérieur, les 


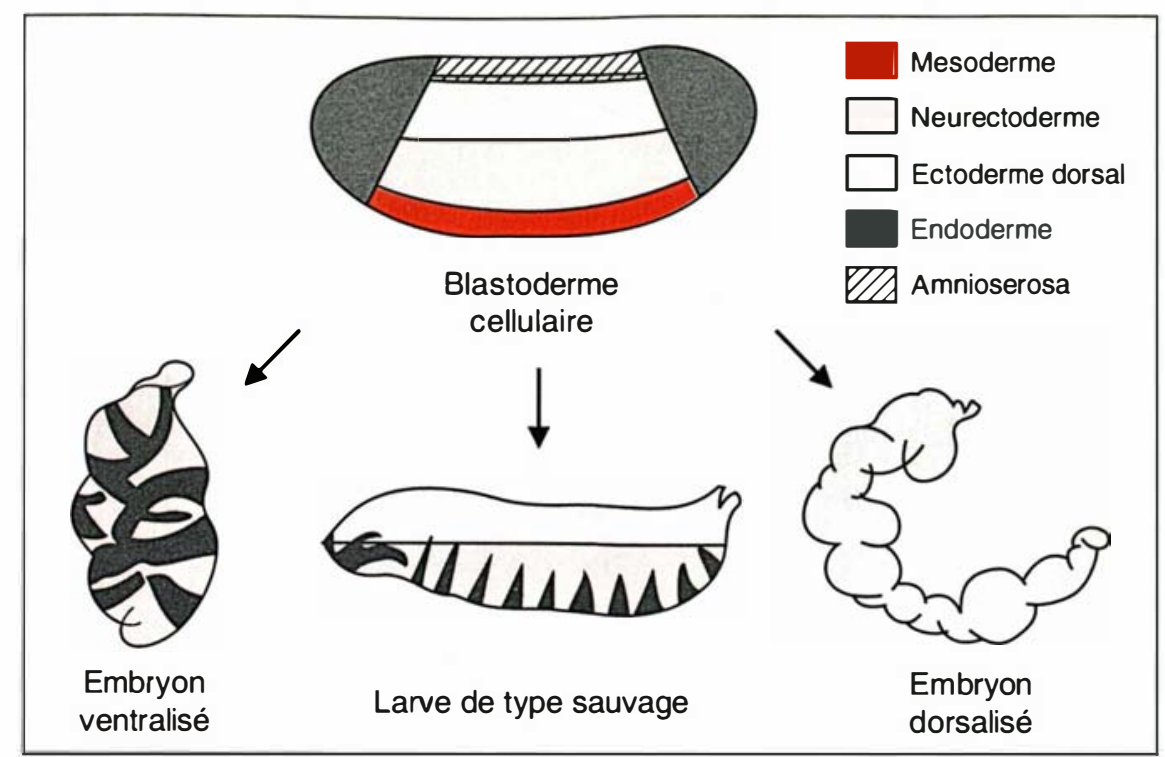

Figure 2. Carte des territoires présomptifs au stade du blastoderme celIulaire et développement larvaire sauvage et mutant. Les bandes de denticules (en noir) constituent à la fois un marqueur de segmentation et un marqueur de la région ventrale (voir texte pour explication).

transcrits du gène bicoid, piégés dès leur entrée dans l'ovocyte au pôle antérieur de la cellule par des protéines spécifiques, constituent l'information de position. Après traduction, la protéine bicoid, par suite d'un mécanisme de diffusion-dégradation, se trouve répartie selon un gradient exponentiel de concentration et, sous cette forme, étant donné la présence d'un homéodomaine, joue le rôle d'un facteur de transcription. Dans ce cas, information de position et facteur de transcription coïncident et sont représentés par différents produits d'un même gène. Notons également que la distribution en gradient est directement dictée par la géométrie de la chambre ovarienne. La situation est plus complexe pour le système postérieur. L'information de position est représentée par le produit du gène nanos localisé à l'extrémité postérieure de l'ovocyte, mais, dans ce cas nanos n'est pas un facteur de transcription, il agit en inhibant hunchback qui, lui-même, est un répresseur de knirps. Ainsi, indirectement, par suite d'une double régulation négative, nanos permet l'expression de knirps. Le troisième système repose sur un mécanisme de transduction de signal qui fait intervenir larve, l'ectoderme ventral (qui, entre temps, aura donné naissance au système nerveux) et l'ectoderme dorsal.

La stratégie pour comprendre la formation de ce plan de développement consiste à rechercher des mutants présentant des altérations de la cuticule chez la larve. Ces modifications traduisent en fait une modification précoce de la carte des territoires présomptifs.

Ces mutations sont de deux types : dorsalisantes, elles consistent en une extension des territoires dorsaux au détriment des territoires ventraux, ce qui, au niveau larvaire, se traduit par la formation d'un tube creux d'ectoderme. Ventralisantes, elles relèvent de la situation inverse, avec déplacement de l'information ventrale vers les régions dorsales, ce qui conduit à l'hyperventralisation de la larve (figure 2). Insistons sur le fait que les gènes correspondants, responsables de la fonction sauvage, auront un effet ventralisant dans le premier cas et dorsalisant dans le second cas.

à la fois la lignée germinale et la lignée somatique; cette fois l'information de position est localisée dans les cellules folliculaires : c'est le produit du gène torsolike. Ce produit interagit avec la protéine torso, protéine transmembranaire à fonction de récepteur tyrosine kinase et l'active spécifiquement aux extrémités de l'ovocyte, permettant ainsi après une série d'étapes intermédiaires non encore identifiées, l'expression des gènes zygotiques tailless et huckebein.

\section{Développement dorso-ventral}

\section{Identification des mutants}

La mise en place du schéma corporel dorso-ventral semble plus simple dans la mesure où elle ne fait intervenir qu'un seul système qui aboutit dès le blastoderme à la définition de cinq territoires : le mésoderme, l'ectoderme ventral (ou neurectoderme), l'ectoderme dorsal, l'amnioserosa (membrane externe de l'œuf) et, aux deux extrémités, l'endoderme (figure 2). Par suite des mouvements morphogénétiques qui accompagnent la gastrulation, endoderme et mésoderme se retrouvent à l'intérieur, si bien que seuls seront visibles sur la
Le Tableau I ( $p$. 658) recense la quasitotalité des mutations connues à ce jour qui affectent la polarité $\mathrm{D} / \mathrm{V}$. Ces mutations sont de deux types: (1) les mutations à effet maternel ; les gènes correspondants s'expriment au cours de l'ovogenèse et son responsables de la mise en place de l'information de position dans l'œuf; (2) les mutations zygotiques; les gènes correspondants constituent les gènes cibles du facteur de transcription représentant l'information de position, à laquelle ils répondent par une expression localisée, définissant ainsi des territoires longitudinaux le long de l'axe dorso-ventral (figure 2). Parmi les mutations à effet maternel, on distingue les mutations qui affectent exclusivement la polarité embryonnaire et les mutations qui, outre la polarité embryonnaire, affectent la polarité de l'œuf. J'y reviendrai plus tard. Pour chacune de ces classes, on connaît des mutations vensantes.

La classe des mutations dites du groupe dorsal (du nom de la première de ces mutations) est la mieux connue. La perte de fonction de l'un quelconque des gènes correspondants tralisantes et des mutations dorsali- 


\section{RÉFÉRENCES}

13. Hashimoto C, Gerttula S, Anderson $\mathrm{KV}$. Plasma membranc localization of the Toll protein in the syncitial Drosophila cmbryo : importance of transmembranc signaling for dorsal-ventral pattern formation. Development 1991;111: 1021-8.

14. Margucric G, Berthicr R, Dupperay A, Hudry-Clergeon G, Uzan G. Les adhésions ccllulaircs, variation sur un thèmc. médecine/sciences $1987 ; 3: 326-33$.

15. Stcin D, Roth S, Vogelsang C, Nüsslcin-Volhard C. The polarity of the dorsoventral axis in the Drosophila embryo is defined by an extracellular signal. Cell $1991 ; 65: 725-35$

16. Wicschaus E, Marsh J, Gehring W. fs(1) K10, a germ-linc dependent femalc stcrilc mutation causling abnormal chorion morphology in Drosophila melanogaster. Arch Dev Biol 1978; 184 : 75-82.

17. Schüpbach T, Germline and soma cooperate during cogencsis to establish the dorso-ventral pattern of egg shell and embryo in Drosophila melanogaster. Cell 1987 ; 49 : 699-707.

18. Hanlin M, Steller H, Pirrotta V, Mohicr E. A 43 kilobase cosmid $P$ transposon rescues the fs(1) K10 morphogenetic locus and threc adjacent drosophila developmental mutants. Cell $1985 ; 40$ : 827-37.

19. Hacnlin M, Ross C, Cassab A, Mohicr E. Oocytc-specific transcription of $\mathrm{fs}(1)$ K10 : a Drosophila genc affecting dorsalventral developmental polarity. $E M B O \mathrm{~J}$ $1987 ; 6: 801-7$

20. Prost E, Deryckere F, Roos C, Hanlin M, Pantesco V, Mohier E. Role of the oocytc nuclcus in detcrmination of the dorsoventral polarity of Drosophila as revealed by molccular analysis of the $K 10$ genc. Genes Dev 1988 ; 2 : 891-900.

21. Montcll D, Keshishian H, Spradling A. Laser ablation studies of the role of the Drosophila oocytc nucleus in pattern formation, Science 1991; 254: 290-3.

22. Price J, Clifford R, Schüpbach T. The maternal ventralizing locus torpedo is allelic to faint little ball, an embryonic lethal, and encodes the Drosophila EGF reccptor homolog. Cell 1989 ; 56 : 1085-92.

23. Schejter E, Shilo BZ. The Drosophila EGF receptor homolog (DER), is allelic to faint little ball, a locus cssential for cmbryonic devclopment. Cell 1989 ; 56 : 1093-104.

24. Gruss P, Kessel M. Axial specification in higher vertcbrates. Curr Op Gen Dev 1991; 1 : 204-10

25. Gurdon JB. The generation of diversity and pattern in animal development. Cell

\begin{tabular}{|c|c|c|c|}
\hline \multicolumn{4}{|c|}{$\begin{array}{c}\text { Tableau I } \\
\text { MUTATIONS AFFECTANT LA POLARITÉ } \\
\text { DORSO-VENTRALE }\end{array}$} \\
\hline \multirow[b]{2}{*}{ Phénotype } & \multicolumn{2}{|c|}{ Mutations à effet maternel affectant } & \multirow{2}{*}{$\begin{array}{l}\text { Mutations } \\
\text { zygotiques }\end{array}$} \\
\hline & $\begin{array}{l}\text { la forme de } \\
\text { l'œuf et le } \\
\text { développement } \\
\text { embryonnaire }\end{array}$ & $\begin{array}{l}\text { le développement } \\
\text { embryonnaire }\end{array}$ & \\
\hline Dorsalisation & $\begin{array}{l}\text { fs }(1) K 10 \text { (motif } \\
\text { hélice-tour-hélice }\end{array}$ & 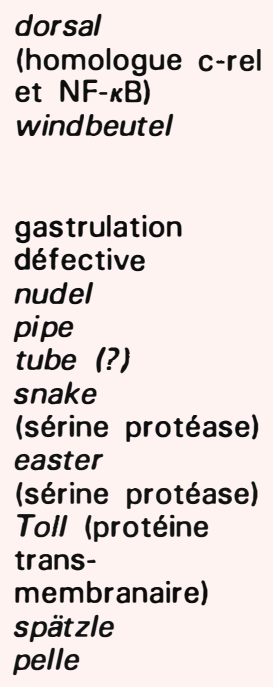 & $\begin{array}{l}\text { snail (motif } \\
\text { "doigts à } \\
\text { zinc ") } \\
\text { twist (motif } \\
\text { hélice-boucle-hé- } \\
\text { lice) }\end{array}$ \\
\hline Ventralisation & $\begin{array}{l}\text { gurken } \\
\text { torpedo } \\
\text { (récepteur } \\
\text { EGF) }\end{array}$ & cactus $(\mathrm{I}-\mathrm{\kappa} \mathrm{B})$ ? & $\begin{array}{l}\text { decapentaplegic } \\
\text { (TGF- } \beta \text { ) } \\
\text { zerknült } \\
\text { (homéo- } \\
\text { domaine) } \\
\text { twisted } \\
\text { gastrulation }\end{array}$ \\
\hline
\end{tabular}

fait apparaître un développement dorsalisé [3]. Une douzième mutation, cactus, aboutit au phénotype inverse, ventralisé [4]. Le spectre continu de phénotypes qui varient en fonction de la force de l'allèle étudié est compatible avec un modèle en gradient, modèle selon lequel la concentration locale d'un morphogène détermine une valeur positionnelle le long de l'axe $\mathrm{D} / \mathrm{V}$. Très tôt, on a donc émis l'hypothèse que le produit de l'un de ces gènes devait être le morphogène $\mathrm{D} / \mathrm{V}$ et que sa répartition en gradient était contrôlée par l'action conjuguée des produits des autres gènes du groupe dorsal [5]. Plusieurs résultats expérimentaux ont suggéré que dorsal était le morphogène ; par exemple, le fait que seule la fonction dorsal soit absolument nécessaire pour la formation de structures ventrales dans les doubles mutants dorsal/cactus. En effet, les embryons produits par des femelles dorsal/cactus sont totalement dorsalisés, tandis qu'en association avec cactus, chacune des autres mutations du groupe dorsal donne naissance à des embryons latéralisés, c'est-à-dire capables de former des structures partiellement ventralisées. Le gène dorsal a été cloné ; au cours des premiers stades du développement, les transcrits, puis la protéine, sont répartis uniformément dans l'œuf; mais, dès la formation du blastoderme syncitial, on voit se former un gradient de concentration nucléaire du produit dorsal (figure 3) [6]. Une observation attentive montre que la quantité globale de protéine est uniforme, seule varie $\mathrm{m} / \mathrm{s} n^{\circ} 7$, vol. 8, seplembre 92 


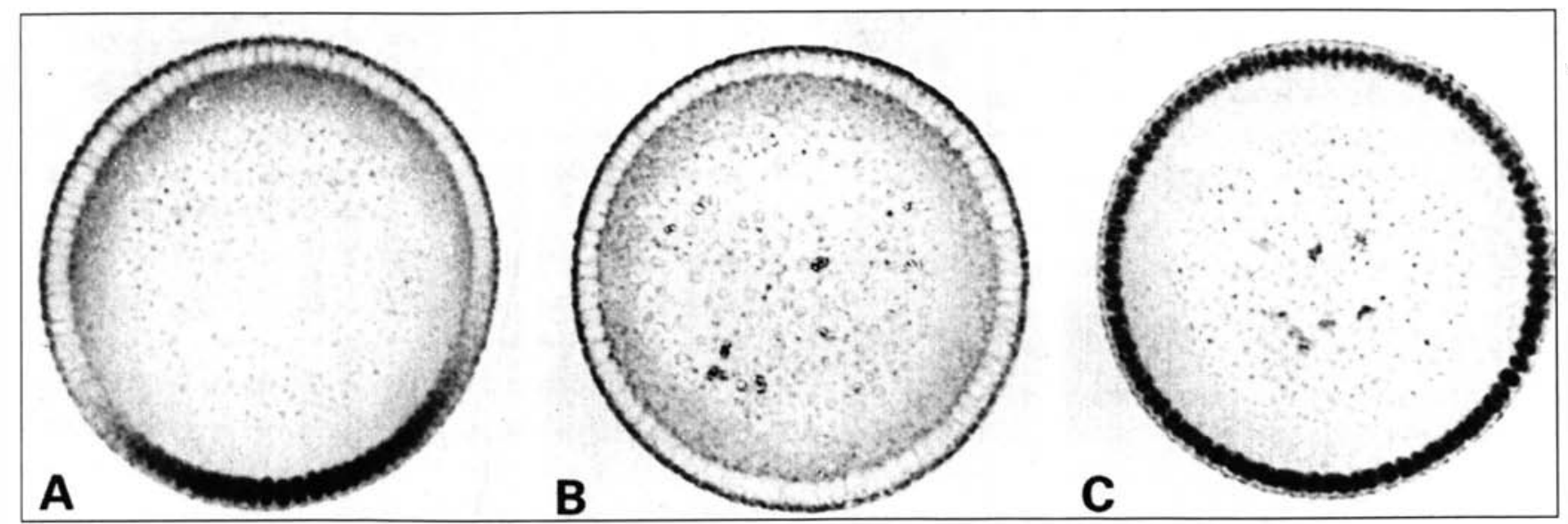

Figure 3. Distribution de la protéine dorsal dans des embryons normaux et mutants au stade blastoderme cellulaire. La protéine est détectée par immunohistochimie. (A) Embryon de type sauvage : la protéine dorsal forme un gradient de concentration nucléaire avec un maximum dans la région ventrale. (B) Embryon dorsalisé (pelle); la protéine est exclusivement présente dans le cytoplasme. (C) Embryon ventralisé ; la protéine est nucléaire sur toute la périphérie de l'embryon. (Cliché reproduit à partir de [1], avec la permission de C. Nüsslein-Volhard.)

sa localisation subcellulaire : totalement nucléaire dans les régions ventrales, elle est également répartie entre cytoplasme et noyau dans les régions latérales, et exclusivement cytoplasmique dans les régions dorsales $[6,7]$. La protéine dorsal, qui présente une forte homologie avec l'oncogène v-rel ainsi qu'avec la sousunité p50 du facteur de transcription $\mathrm{NF}-\kappa \mathrm{B}$, assume elle-même la fonction $\mathrm{du}$ facteur de transcription $\left(\mathrm{m} / \mathrm{s} n^{\circ} 1\right.$, vol. 7, p. 67). On conçoit, dans ces conditions, l'importance de sa localisation nucléaire. Dans les mutants dorsalisants, la protéine dorsal est présente mais entièrement cytoplasmique, contrairement aux embryons cactus (ventralisés) où elle est exclusivement nucléaire (figure 3 ), ce qui suggère que la fonction des gènes du groupe dorsal serait de faciliter la localisation nucléaire de dorsal, tandis que cactus l'inhiberait. Notons, par ailleurs, qu'il a été possible d'établir une corrélation entre la quantité de produit dorsal nucléaire et le type de cuticule formé chez le mutant ou, ce qui revient au même, l'expression de ses gènes cibles, twist et zerknült ; c'est bien là le comportement attendu pour un morphogène.

L'antagonisme existant entre dorsal et cactus rappelle l'interaction $\mathrm{NF}-\kappa \mathrm{B}$ et
$\mathrm{I} \kappa \mathrm{B}$. L'association de NF- $k \mathrm{~B}$ avec $\mathrm{I} \kappa \mathrm{B}$ inhibe la localisation nucléaire de NF$\kappa \mathrm{B}$, tandis que la phosphorylation de $\mathrm{I} \kappa \mathrm{B}$ est nécessaire pour permettre la dissociation du complexe et l'activation de NF- $\kappa$ B [9]. Bien que l'identification moléculaire n'ait pas encore pu être établie, tout porte à croire que cactus serait l'homologue de $\mathrm{I} \kappa \mathrm{B}$. Comment le gradient nucléaire dorsal est-il établi ?

Nous avons vu que, pour l'axe A/P, la mise en place du gradient bicoid était dictée par la polarité $\mathrm{A} / \mathrm{P}$ de la chambre ovarienne. La situation n'est pas aussi simple pour le système $\mathrm{D} / \mathrm{V}$ dans la mesure où aucune asymétrie $\mathrm{D} / \mathrm{V}$ n'est clairement visible dans la chambre ovarienne.

Un certain nombre d'informations ont été obtenues à partir des expériences de transplantation de cytoplasme. Ces expériences, qui consistent à prélever une très petite quantité de cytoplasme chez un embryon donneur, mutant ou non, et à l'injecter à un embryon receveur, permettent de tester la " capacité de sauvetage" présente dans le cytoplasme injecté, sauvetage qui se manifestera par l'apparition de structures cuticulaires ventrales $[10$, 3]. Au cours de telles expériences, on a pu constater que le cytoplasme d'un embryon de type sauvage était capa- ble de sauver tous les mutants à l'exception de windbeutel, pipe et nudel; plus intéressant, le cytoplasme de chaque mutant est capable de sauver tous les autres mutants, ce qui indique que la fonction mutée chez le donneur n'est pas nécessaire à l'expression de la fonction mutée du receveur ; enfin, quelque soit le site d'injection du cytoplasme, les structures ventrales apparaissent toujours dans la région ventrale de l'œuf. Cela démontre la présence d'une polarité résiduelle de l'embryon par rapport à la polarité de l'œuf et qui fait que, même chez un embryon ayant perdu la capacité de différencier des structures ventrales, le dos et le ventre ne sont pas équivalents.

Seule exception à cette règle, Toll : si l'on injecte du cytoplasme sauvage à des embryons Toll $^{-}$, les structures ventrales se forment toujours au site d'injection [11] ; l'embryon Toll- a perdu sa polarité intrinsèque, ce qui signifie qu'il n'existe plus de lien entre la forme de l'œuf et la polarité embryonnaire ; par conséquent, on est en droit de penser que c'est le produit du gène Toll qui assure ce lien. Le gène Toll a été cloné, il code pour une protéine transmembranaire répartie uniformément sur toute la membrane de l'œuf $[12,13]$. Le 
domainc extracellulaire est similaire à la chaîne $\alpha$ de la glycoprotéine I/GPIb, qui intervient dans la coagulation du sang et qui scrt de récepteur à la thrombinc et au facteur de von Willebrand [14], tandis que la région intracellulairc est homologue au récepteur de l'interleukine 1 (IL-

1) ; or, on sait que dans les lymphocytes, unc chaîne de réactions initiée par le récepteur de l'IL-1 a pour effet d'activer la translocation nucléairc du factcur de transcription $\mathrm{NF}-\kappa \mathrm{B}$. Lorsque l'on se souvient que dorsal est homologue à une sous-unité de $\mathrm{NF}-\kappa \mathrm{K}$, on se retrouve dans unc situation analoguc où Toll participerait à la translocation nucléaire de dorsal.

Revenons au domaine extracellulaire de la protéine Toll similaire à la chaînc $\alpha$ de GPIb, récepteur de la thrombine. Un raisonnement par analogie avec ce qui se passe dans le système terminal de l'axe $\mathrm{A} / \mathrm{P}$, où le récepteur tyrosine kinase torso a pour ligand le produit torsolike synthétisé par les cellules folliculaires, suggère que le ligand de Toll pourrait également être synthétisé par les ccllules folliculaires. Comme la protéine torso, nous avons vu que la protéine Toll était répartic uniformément sur toute la membrane de l'œuf. Si le ligand de Toll cst produit de façon asymétrique par les cellules folliculaires de la région ventrale de l'ovocyte, il y aura activation sélective de Toll dans cette région ventrale de l'ovocyte, ce qui aurait pour conséquence la translocation nucléaire de dorsal spécifiquement dans la région ventrale de l'œuf.

Récemment D. Stcin et al., dans le laboratoire de C. Nüsslein-Volhard, ont réalisé une sćrie d'expériences très élégantes qui ont permis de confirmer ces hypothèses [15]. Tout d'abord, ils ont montré, par la construction de mosaïques entre lignée germinalc et lignée somatique, que les mutations windbeutel, pipe et nudel étaient dépendantes de la lignée somatique et que, par conséquent, les gènes correspondants devaient s'exprimer dans les cellules folliculaires. Il était alors concevable que le produit de l'un au moins de ces gènes fût le ligand de Toll et que si tel était le cas, il dût être sécrété dans l'espace périvitellin (espace défini par la membrane cytoplasmique de l'ovocyte et la membrane vitclline synthétisće par les cellules folliculaires). L'expérience a alors consisté à prélever une petite quantité de liquide périvitcllin et à l'injecter à des embryons mutants windbeutel, pipe ct nudel. D. Stcin a montré qu'il était possible de sauver partiellement ces mutants ct que le sauvetage avait licu au site d'injection [15]. Cette expérience exige toutefois que le liquide périvitellin soit prélevé sur des embryons Toll-. Cette condition préalable confirme que l'activité de sauvetage pourrait bien représenter le ligand de Toll puisqu'clle est présente sous une forme libre dans des embryons Toll $^{-}$. En revanche, si techniquement, l'utilisation d'embryons Toll- ${ }^{-}$dorsalisés facilite la délicate opération du prélèvement de liquide périvitellin, elle interdit pour l'instant toute déduction quant à l'éventuelle localisation spécifique du ligand sur la face ventrale de l'œuf, les cmbryons Toll $^{-}$, dorsalisés, ayant, par définition, perdu toutc polarité dorso-ventrale.

Ces résultats, ainsi que ceux obtenus pour le système responsable des extrémités pour l'axe $\mathrm{A} / \mathrm{P}$, mettent en évidence la contribution des cellules folliculaires dans l'établissement de la polarité embryonnaire. De plus, en démontrant (pour l'axe $A / P$ ), et en suggérant (pour l'axe $\mathrm{D} / \mathrm{V}$ ), que cette participation des cellules folliculaires s'effectue sclon un schéma asymétrique, ces données impliquent que la chambre ovarienne est polarisée, et ccla non seulement selon l'axe A/P, mais aussi sclon l'axc D/V. Ainsi, la polarité embryonnaire ne serait que la conséquence d'une polarité précxistante de la chambre ovarienne, polarité dont il s'agit maintenant de comprendre l'origine.

\section{Mécanismes de la polarité de la chambre ovarienne}

Un autre argument en faveur du rôle des ccllules folliculaircs dans l'établissement de la polarité $\mathrm{D} / \mathrm{V}$ repose sur l'existence d'un petit groupe de mutations qui affectent à la fois la polarité embryonnaire et la polarité de l'œuf, démontrant par là-même qu'il existe une étape du développement au cours de laquelle les deux polarités, de l'œuf et de l'embryon, sont couplécs. Ce petit groupe de mutations comprend les trois mutations $f s(1) K 10[16]$, gurken et torpedo [17].

Les œufs pondus par des femelles homozygotes $K 10$ sont cylindriques, et les appendices respiratoires, normalement implantés dorsalement, entourent l'extrémité de l'œuf, traduisant ainsi son caractère dorsalisé. Ces œufs donnent naissance à des embryons dorsalisés. Cette mutation, par ailleurs sans effet sur la viabilité des mouches, est strictement dépendante de la lignée germinale [16]. Cette apparente contradiction entre un gène s'exprimant dans la lignée germinale, mais dont la perte de fonction conduit paradoxalement à un défaut du chorion, a constitué la première mise en évidence d'un transfert d'information entre la lignée germinale et la lignée somatique au cours de l'ovogenèse.

Le gènc $K 10$ a été cloné [18]. L'hybridation in situ de sondes radioactives ou froides sur des coupes d'ovaires a montré que les transcrits étaient exclusivement présents dans l'ovocyte ct, plus particulièrement, dans la région proche du noyau, ce qui suggère qu'ils sont effectivement synthétisés dans l'ovocyte [19]. Cette expression est en accord avec le caractère "germinaldépendant " de la mutation mais contredit l'idée généralement admise de l'inactivité transcriptionnelle de l'ovocyte. Cette spécificité d'expression, qui semble propre à $K 10$, mais dont on ignore encore l'origine et la signification biologique, se retrouve au niveau protéique, puisque la protéine K10 révélée par immunohistochimie est exclusivement détectée dans le noyau de l'ovocyte [20]. Il faut noter que ce gène s'exprime également à tous les stades du développement, y compris chez le mâle. Cependant, cette expression tardive n'a pu être reliée à aucune fonction précise étant donné l'absence de tout phénotype en dehors de la stérilité femelle. Par ailleurs, la séquence ayant montré la présence d'un motif " hélicc-tour-hélice " caractéristique des protéines se liant à l'ADN, il scmble raisonnable de penser que $K 10$ possède une fonction régulatrice, fonction qui, logiquement, doit s'exercer au niveau de l'ovocyte. 
C'est ainsi qu'indirectement l'étude moléculaire du gène $K 10$ a montré le rôle du noyau de l'ovocyte dans le déterminisme dorso-ventral de la chambre ovarienne.

L'observation des mouvements morphogénétiques qui accompagnent la maturation de l'ovocyte met en évidence la fonction dorsalisante du noyau de l'ovocyte ; en effet, dès le début de la vitellogenèse (stade 8 ), le noyau de l'ovocyte adopte une position excentrée au pôle antétieur de l'ovocyte, proche des cellules folliculaires. Au cours des stades suivants, les cellules folliculaires situées à proximité du noyau vont migrer et s'accumuler entre l'ovocyte et les cellules nourricières; plus tard au cours de l'ovogenèse, on constate que ce sont ces mêmes cellules qui synthétiseront les appendices respiratoires de l'ovocyte. Cette corrélation entre la localisation du noyau et la formation des appendices dorsaux a pu être confirmée par l'observation de la mutation dicephalic. Certaines chambres dicephalic ont la particularité de présenter un ovocyte en position centrale avec des cellules nourricières réparties aux deux extrémités. Cette anomalie s'accompagne d'une délocalisation du noyau de l'ovocyte, qui peut indifféremment occuper une position antérieure ou postérieure dans l'ovocyte. On a pu constater qu'il y avait, dans tous les cas, coïncidence entre la position du noyau et la localisation des futurs appendices dorsaux.

Récemment, la fonction dorsalisante du noyau a été directement mise en évidence en montrant que sa destruction par un rayonnement laser aboutit à la formation d'ovocytes ventralisés [21]. Il est raisonnable de penser que cette fonction dorsalisante puisse s'exercer par l'intermédiaire d'une substance synthétisée à partir du noyau de l'ovocyte, et qui diffuserait dans le cytoplasme pour atteindre en priorité les cellules folliculaires les plus proches auxquelles elle transmettrait une information dorsalisante. Étant donné les propriétés de la protéine et le phénotype dorsalisé qui accompagne la mutation, il est peu vraisemblable que $K 10$ puisse assumer la fonction de facteur diffusible. On s'attend, de plus que la perte du signal dorsalisant s'accom- pagne des mêmes effets que ceux résultant de l'ablation du noyau, c'est-à-dire une ventralisation.

De là, l'intérêt pour deux mutations aux effets opposés à ceux décrits pour K10 : les mutations gurken et torpedo isolées par T. Schüpbach [17]. Comme $K 10$, ces deux mutations affectent à la fois la forme de l'œuf et le développement embryonnaire, mais, contrairement à $K 10$, leur effet est ventralisant (figure 4). Les œufs sont très allongés, la ventralisation se manifeste par la fusion voire la disparition des appendices et, au niveau embryonnaire, par une expansion des territoires ventraux, essentiellement le mésoderme, qui va envahir tout

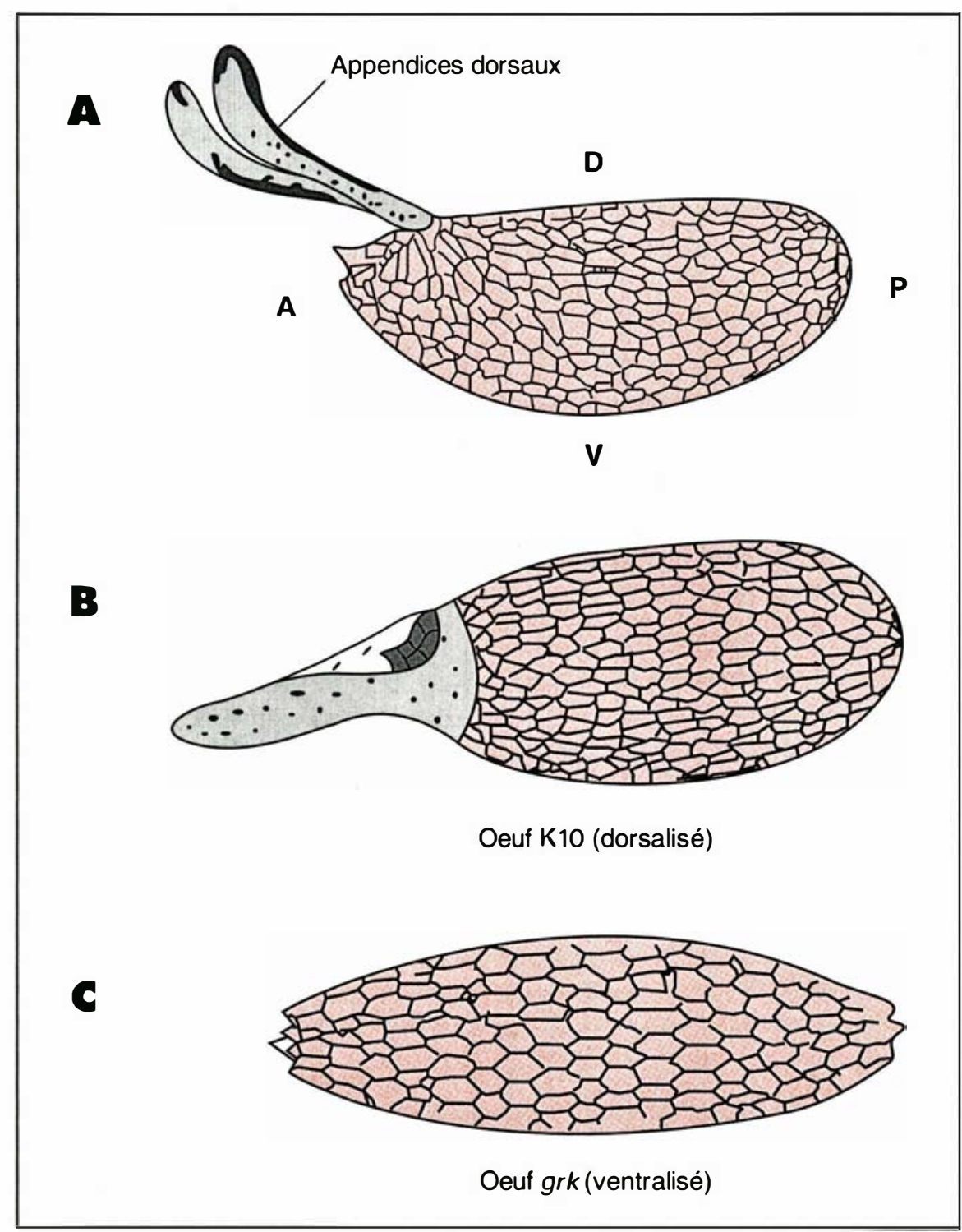

Figure 4. Représentation semi-schématique des ceufs de drosophile. (A) CEuf pondu par une femelle de type sauvage. L'asymétrie A/P et $D / V$ est évidente; noter l'implantation antéro-dorsale des appendices respiratoires. (B) OEuf pondu par une femelle $\mathrm{K} 10$. L'cuf a perdu son asymétrie $D / V$, il est cyclindrique par rapport à l'axe $A / P$ et les appendices entourent toute l'extrémité antérieure. (C) CEuf pondu par une femelle gurken. Les appendices dorsaux ont disparu, l'œuf est très allongé, aucune polarité D/V n'est détectée. Le réseau qui apparaît à la surface de l'œuf représente l'empreinte laissée par les cellules folliculaires sur le chorion, sa forme constitue un marqueur de polarité $D / V$ du chorion. 
l'embryon au détriment des structures dorsales et dorso-latéralcs. Les phénotypes de ces deux mutations sont très proches ; la différence principale réside dans le fait que gurken dépend de la lignée germinale et torpedo de la lignée somatique. Le caractère " germinal-dépendant " de gurken ne fait que confirmer l'idée d'un transfert d'information de la lignéc germinale, vraisemblablement l'ovocyte, vers la lignée somatique. Étant donné les similitudes existant entre les deux mutations, il est logique de penser que les deux gènes $K 10$ et gurken sont impliqués dans une voie de développement commune, qui pourrait être la production et la répartition du signal dorsalisant. En revanche, le caractère "somatiquedépendant " de torpedo suggère que cette mutation empêche les cellules folliculaires de percevoir le signal dorsalisant. En outre, le fait que torpedo affecte également le développement de l'embryon démontre l'influence, directe ou indirecte, des cellules folliculaires sur la mise en place de la polarité embyronnairc. Ainsi, ces observations démontrent que l'interaction entre lignéc germinalc et lignée somatique est nécessaire non sculcment pour l'établissement de la polarité de la chambre ovarienne, mais aussi pour la polarité de l'embryon.

Le gène torpedo a été cloné de façon fortuite et identifié comme le récepteur EGF de la drosophile (DER) [22, 23]. L'hybridation in situ montre que les transcrits sont uniformément distribués dans toutes les cellules folliculaires qui entourent l'ovocytc. En revanche, aucun signal n'est détecté dans l'ovocyte ou dans les cellules nourricières, confirmant bien le caractère somatique de la mutation. Par ailleurs, on a pu montrer que ce gène avait également une expression zygotique qui, lorsqu'elle est altérée, conduit à une létalité embyronnaire. Une analyse génétique plus approfondie a conclu à l'identité de torpedo ct de faint little ball, dont le caractère pléiotropique répond à la nature multifonctionnellc d'un réceptcur EGF impliqué dans de nombreux processus biologiques [22, 23]. La similitude des effets observés pour les trois mutations (même s'ils sont antagonistes) suggère que les gèncs interviennent dans un processus commun. Cettc interaction a été confirméc par la misc en évidence d'une relation d'épistasic de chacune des mutations ventralisantes sur la mutation $K 10$. Les femelles homozygotes pour $K 10$ et torpedo ou $K 10$ et gurken produisent des œufs ventralisés à l'intéricur desquels se développent des embryons également ventralisés. De plus, nous avons pu montrer que dans la combinaison $K 10 / K 10$; gur$k e n /+$, gurken se comportait comme une suppresseur partiel dominant de $K 10$; cette caractéristique propre à gurken suggère une relation privilégiée entre les deux gènes.

L'cnsemble de ces données est compatible avec un modèle selon lequel le signal dorsalisant serait le produit du gène gurken. L'expression de ce gène serait contrôlée négativement par K10. Le produit gurken diffuserait dans le cytoplasme et agirait par l'intermédiaire de torpedo sur les cellules folliculaires leur conférant une identité dorsalisée.

La technique ditc de "piège à enhancer " (enhancer trap) $\left(\mathrm{m} / \mathrm{s} n^{\circ} 3\right.$, vol. 8, p. 268) - qui, chez la drosophile, permet de visualiser le schéma d'expression de gènes grâce à l'insertion au hasard d'un transposon $P$ couplé à un gène rapporteur, la $\beta$ galactosidase a montré qu'il existait un certain nombre de gènes dont le profil d'expression selon un double gradient $\mathrm{A} / \mathrm{P}$ et $\mathrm{D} / \mathrm{V}$ centré sur le noyau de l'ovocyte pourrait figurer cette réponse des cellules folliculaires à un signal émis à partir du noyau de l'ovocyte. Il est vraisemblable toutefois que ce modèlc soit trop schématique ; il cst possible, en effet, que d'autres gènes non encore identifiés participent de façon directe ou indirecte à la mise en place de la polarité de la chambre ovarienne. Par ailleurs, le modèle énoncé ne tient pas compte de la structure interne de l'ovocyte, il est pourtant évident que certains constituants du cytosquelette doivent jouer un rôle dans la diffusion du signal. Quand à l'identification du signal, il faut attendre l'analyse moléculaire du gène gurken pour apporter une réponse définitive. Un résultat récent vient toutefois confirmer le modèlc proposé pour les interactions entre $K 10$, gurken et torpedo. Nous avons pu cn effet montrer que l'cxpression du gènc $K 10$ était soumise à un mécanisme d'autorégulation ; le site de fixation de la protéine ayant été localisé dans la région 3' de l'intron. Or, l'étude des séquences régulatrices du gène par la technique de la transformation à l'aide de l'élément $\mathrm{P}$ a montré que le gène $K 10$ dépourvu d'intron conservait sa fonction sauvage dans la mesurc où les femelles mutantes $K 10$ porteuses d'un transposon $K 10$ sans intron pondent des œufs de type sauvage qui, dans leur majorité, donnent naissance à des embryons sauvages, avec toutefois une proportion significative (10 à $15 \%$ ) d'embryons ventralisés.

Ce résultat corrobore l'idée selon laquclle $K 10$ se comporte comme un régulateur négatif du signal dorsalisant. En effet, la surproduction de $K 10$ consécutive à l'absence de régulation donne lieu à un phénotype analogue à celui qui résulte d'une absence de signal dorsalisant ; c'cstà-dire un dévcloppement ventralisé.

\section{Conclusion}

La drosophile est à l'heure actuelle celui des organismes eucaryotes dont le développement précoce est le mieux connu; ce succès doit beaucoup à l'utilisation combinée de la génétique et de la biologie moléculaire qui ont permis de préciser des notions déjà anciennes d'information de position, de gradient de morphogène et de leur donner un support concret au niveau moléculaire. Cela constitue sans doute l'une des plus bclles réussites de la biologie de ces dernières années. Au cours de ces études, la drosophile est apparue non sculement comme un modèle, mais surtout comme un point de départ pour l'étude du développement des organismes supérieurs, ne serait-ce qu'en fournissant des sondes de gènes clés inaccessibles autrement chez les vertébrés par les techniques classiques. On sait à cet égard quelle a été la contribution de la drosophile à la compréhension du développement de la souris $[24,25]$.

Même s'il apparaît claircment que le modèle élaboré pour la drosophile ne peut s'adapter globalement aux autres organismes étant donné certaines modalités de développement propres 
à la drosophile (les principales étant que les premières étapes se déroulent dans un syncitium et que le développement débute dans un ovocyte qui possède déjà une polarité), il n'en reste pas moins vrai que nombre d'étapes individuelles peuvent être intégralement transposées chez les vertébrés supérieurs, ainsi pour la localisation des transcrits, la transduction de signaux, le contrôle de l'expression génique et la régulation de la translocation nucléaire d'un facteur de transcription; notons le cas de la protéine Toll, particulièrement intéressant au regard de l'évolution puisque s'y trouvent associées, respectivement dans les domaines extracellulaire et cytoplasmique, deux fonctions qui, chez les vertébrés, sont séparées et qui participent à des chaînes biologiques différentes, l'une dans la coagulation du sang, l'autre dans l'activation des lymphocytes mettant en jeu, notamment, la translocation nucléaire du facteur NF- $\mathrm{BB}$.

En dépit de quelques points encore obscurs, les premières étapes du développement, celles qui vont de l'œuf pondu à la réalisation du plan d'organisation de l'embryon, sont bien connues ; en revanche, on connaît mal la façon dont l'organisme adulte ćlabore un œuf, en particulier, la façon dont il lui transmet sa polarité. Si la définition de la polarité A/P semble relativement simple puisqu'elle tire son origine de la configuration spatiale des cellules nourricières et de l'ovocyte, configuration qui apparaît dès la différenciation de l'ovocyte, il n'en va pas de même pour la polarité $\mathrm{D} / \mathrm{V}$ pour laquelle il n'existe pas d'élément d'asymétrie avant le stade 8 de l'ovogenèse, stade au cours duquel le noyau de l'ovocyte adopte une localisation antéro-dorsale. le noyau de l'ovocyte constitue-t-il la seule source de polarité $\mathrm{D} / \mathrm{V}$ de la chambre ovarienne ? Nous avons vu que les cellules folliculaires jouaient un rôle décisif dans la polarité $\mathrm{D} / \mathrm{V}$, non seulement par l'intermédiaire de gènes tels que torpedo dont la fonction peut être directement soumise à l'influence du noyau de l'ovocyte, mais également par l'intermédiaire de gènes d'expression somatique tels que windbeutel, pipe ou nudel. Doit-on voir dans la fonction de ces gènes la conséquence de l'expression (ou plutôt de la non-expression) d'une fonction dorsalisante émise à partir du noyau de l'ovocyte, ou bien l'un d'entre eux au moins intervient-il de façon autonome, démontrant ainsi l'existence d'un second système de mise en place de la polarité de la chambre ovarienne ? Ce problème, qui revient à se demander comment l'information dorsalisante émise à partir du noyau de l'ovocyte peut être convertie en une information ventralisante dans l'embryon, constitue à l'heure actuelle l'un des nouveaux axes de recherche des spécialistes du développement

\section{TIRÉS A PART}

E. Mohier.

\section{Summary \\ Determination of the dorsoventral polarity of Drosophila}

Embryonic pattern formation has been studied extensively in many organisms. In Drosophila, the powerful combination of genetic, cytoplasm transplantation experiments, as well as recent molecular data, have helped to elucidate the mechanisms responsible for the establishment of embryonic polarity. A small number of genes, most of them maternally expressed, are involved in this process and participate in four independent systems - 3 for the antero-posterior axis (A/P) and one for dorsoventral axis $(\mathrm{D} / \mathrm{V})$ which define various embryonic territories by specifically localized cues. This review concerns the definition of the dorsoventral polarity responsible for the establishment of the germ layers of the embryo. Dorsoventral development is regulated by a single group of maternally expressed genes : the "dorsal group" of genes. It includes 11 genes, the loss of function of any of which results in a dorsalized development, whereas mutation of the 12 th gene, cactus, results in a ventralized development. These genes are arranged according to a functional hierarchy, and have been shown to cooperate in the formation of a graded nuclear concentration of the dorsal gene product. The dorsal product corresponds to the dorsoventral morphogen and is homologous to the transcription factor NF- $k$ B. Among the 11 genes of the dorsal group, 3 are required in the somatic line. This suggests the existence of inductive signals originating during oogenesis from the follicle cells that surround the developing oocyte. This somatically expressed spatial information probably controls dorsoventral development by defining the polarity of a signal transducing pathway that specifically activates the nuclear uptake of the dorsal product. This model, highlights the importance of the polarity of the egg chamber, and suggests that it is the oocyte nucleus, due to its asymmetrical localization, that determines the dorsoventral pattern formation of the embryo. 